\title{
Basispflege lindert Nebenwirkungen der Medikation
}

\begin{abstract}
Akne ist meist gut sichtbar im Gesicht und am Stamm lokalisiert und erzeugt daher einen hohen Leidensdruck. Dies gilt nicht nur für die häufig betroffenen Jugendlichen, sondern zum Beispiel auch für Frauen mit Acne tarda, die oft im mittleren Lebensalter (zwischen 25 und 40 Jahren) auftritt. Vor allem unter den bleibenden Narben und Hyperpigmentierungen leiden die Betroffenen ein Leben lang. „Daher besteht das Therapieziel nicht nur darin, den akuten Zustand zu bessern, sondern die Narbenbildung langfristig zu vermeiden“, erklärte Dr. Markus Reinholz, München. Da die Nar-
\end{abstract}

benbildung früh im Krankheitsverlauf auftritt, spricht sich Reinholz für den frühzeitigen Einsatz einer effektiven Therapie in Form oraler Antibiotika aus.

Für eine Besserung der Symptome kann es bei mild ausgeprägter Akne ausreichen, die Haut $\mathrm{zu}$ reinigen und mit einer Basispflege zu versorgen. In schwereren Fällen ist die Reinigung und Pflege begleitend zur Therapie ebenfalls erforderlich. Denn als Nebenwirkungen einer Medikation tritt häufig eine sehr trockene Haut auf, die Patienten leiden unter starkem Spannungsgefühl. Eine adäquate Basispflege trägt hier wesentlich zur Compliance der Patienten bei. Reinholz rät zu pHneutralen Syndets und einer nicht komedogenen Pflege. Dafür stehen etwa die Produkte der neuen Dermopure-Serie zur Verfügung. Eine zweiwöchige Anwendung von Eucerin ${ }^{\circledR}$ Dermopure Therapiebegleitende Feuchtigkeitspflege bewirkte eine signifikante Verminderung der Hauttrockenheit, Rötung und Rauigkeit [Beiersdorf-interne Studien]. Bei $40 \%$ der Patienten ging das bessere Hautgefühl mit einer klinisch relevanten Verbesserung der Lebensqualität einher. Dr. Marion Hofmann-Aßmus

Symposium "Neueste Pathogenese \& innovative Therapie bei der jugendlichen Akne", anlässlich des 113. Kongresses für Kinder- und Jugendmedizin 2017, Köln, 23. September 2017; Veranstalter: Professor Paul Gerson Unna Akademie (Beiersdorf)

\section{Narben wirksam behandeln}

Neuere wissenschaftliche Übersichtsarbeiten zur Prävention und Behandlung hypertropher Narben zeigen, dass überschießende Narben auch heute noch ein unterschätztes Risiko darstellen. Demzufolge gibt es eine Reihe von Risikofaktoren für die Entwicklung hypertropher Narben wie verzögerte Wundheilung (länger als 21 Tage), entzündliche Erkrankungen der Haut, starke Zugkräfte im Narbenbereich sowie eine genetische
Prädisposition. Weiterhin spielen bei der Ausbildung hypertropher Narben die Topografie und hormonelle Faktoren eine Rolle.

In einer umfangreichen Präventionsstudie [Signorini et al. Aesthetic Plast Surg. 2007;31:183-7] konnte unter anderem gezeigt werden, dass die Wahrscheinlichkeit der Ausbildung einer ästhetisch schönen Narbe um ein Vielfaches höher ist, wenn Narben präventiv mit Kelo-cote ${ }^{\circledast}$ behandelt werden. Daher sollte nach einem chirurgischen Eingriff, nach vollständigem Wundverschluss oder nach Fadenzug, die Behandlung mit dem Silikongel Kelo-cote ${ }^{\circledast}$ beginnen.

Kelo-cote ${ }^{\circledast}$ sollte zweimal täglich hauchdünn aufgetragen werden. Das transparente und geruchlose Gel ist an allen Körperstellen (außer Schleimhäuten) anwendbar. Durch seine hohe Verträglichkeit ist es auch zur Anwendung bei Kindern und auf empfindlicher Haut geeignet.

Nach Informationen von Alliance

Pharmaceuticals

\section{Hautschutz im Herbst}

Bunte Blätter, kräftiger Wind, zunehmende Kälte - im Herbst und Winter benötigt unsere Haut eine wohltuende, schützende Pflege.

Die Dermasence Adtop Creme pflegt gereizte oder trockene bis sehr trockene und sogar zu Neurodermitis neigende Haut mit Ölkomponenten und Glycerin. Dank ihrer besonderen Formulierung bildet sie einen wirkungsvollen hautverwandten Schutzfilm, der atmungsaktiv ist und Feuchtigkeit bindet. So glättet sie raue Haut und macht sie geschmeidig, Spannungsgefühle und Trockenheitsfältchen verschwinden. Zusätzlich wirkt das enthaltene Bisabolol beruhigend. Das Reizpotenzial der Haut wird verringert. Bei täglicher Anwendung stärkt die Creme nachhaltig die Barrierefunktion der Haut.

Die Dermasence Cream + Mask mit ungesättigten Fettsäuren, Hyaluronsäure und einem Vitaminkomplex ist eine intensive Gesichtspflege bei trockener, feuchtigkeitsarmer und empfindlicher Haut. Das enthaltene Nachtkerzensamenöl bindet Feuchtigkeit in der Haut und verringert ihren transepidermalen Wasserverlust (TEWL). Hyaluronsäure liefert und speichert zusätzlich Feuchtigkeit. Raue Haut wird durch das enthaltene Jojobaöl geschmeidig-zart. Zusätzlich wird die Haut durch den Vitaminkomplex aus den Vitaminen C, E, Provitamin B5 und Provitamin A optimal versorgt und vor Umwelteinflüssen geschützt.

Nach Informationen von P\&M Cosmetics 